\title{
The Cholegas trial: long-term results of prophylactic cholecystectomy during gastrectomy for cancer-a randomized-controlled trial
}

\author{
Lapo Bencini ${ }^{1}[$ ( $)$ Alberto Marchet ${ }^{2} \cdot$ Sergio Alfieri $^{3} \cdot$ Fausto Rosa $^{3} \cdot$ Giuseppe Verlato $^{4} \cdot$ Daniele Marrelli $^{5}$. \\ Franco Roviello ${ }^{5}$. Fabio Pacelli ${ }^{6}$. Luigi Cristadoro ${ }^{7}$. Antonio Taddei ${ }^{8} \cdot$ Marco Farsi $^{1} \cdot$ Italian Research Group for $^{4}$ \\ Gastric Cancer (GIRCG)
}

Received: 13 March 2018 / Accepted: 18 September 2018 / Published online: 22 September 2018

(c) The International Gastric Cancer Association and The Japanese Gastric Cancer Association 2018

\begin{abstract}
Background The incidence of cholelithiasis has been shown to be higher for patients after gastrectomy than for the general population, due to vagal branch damage and gastrointestinal reconstruction. The aim of this trial was to evaluate the need for routine concomitant prophylactic cholecystectomy (PC) during gastrectomy for cancer.

Methods A multicenter, randomized, controlled trial was conducted between November 2008 and March 2017. Of the total 130 included patients, 65 underwent PC and 65 underwent standard gastric surgery only for curable cancers. The primary endpoint was cholelithiasis-free survival after gastrectomy for gastric adenocarcinoma. Cholelithiasis was detected by ultrasound exam.

Results After a median follow-up of 62 months, eight patients (12.3\%) in the control group developed biliary abnormalities (four cases of gallbladder calculi and four cases of biliary sludge), with only three (4.6\%) being clinically relevant (two cholecystectomies needed, one acute pancreatitis). One patient in the PC group had asymptomatic biliary dilatation during sonography after surgery. The cholelithiasis-free survival did not show statistical significance between the two groups $(P=0.267)$. The number needed to treat with PC to avoid reoperation for cholelithiasis was 1:32.5.

Conclusions Concomitant PC during gastric surgery for malignancies, although reducing the absolute number of biliary abnormalities, has no significant impact on the natural course of patients.
\end{abstract}

Keywords Prophylactic cholecystectomy · Gastric surgery · Gastric cancer

The members of the "Italian Research Group for Gastric Cancer (GIRCG)" are listed in the acknowledgements section.

Lapo Bencini

lapbenc@tin.it

1 Division of Oncologic Surgery and Robotics, Department of Oncology, Careggi University Hospital, Largo Brambilla 3, 50134 Florence, Italy

2 Department of Surgery, University Hospital, Padua, Italy

3 Digestive Surgery of University Hospital "A. Gemelli”, Rome, Italy

4 Unit of Epidemiology and Medical Statistics, University of Verona, Verona, Italy

5 Department of Surgery, University of Siena, Siena, Italy

6 Surgical Oncology Catholic University, Campobasso, Italy

7 General Surgery, "C. Poma” Hospital, Pieve di Coriano, Mantua, Italy

8 General Surgery, Careggi University Hospital, Florence, Italy

\section{Introduction}

There is long-standing debate regarding the need for concomitant prophylactic cholecystectomy (PC) during gastric surgery because of the supposed subsequent higher incidence of gallstones and gallbladder sludge. Some studies have reported an incidence of gallstones up to $25 \%$ in those patients who have undergone gastric surgery [1-5]. The mechanism of gallbladder impairment is thought to be multifactorial. First, there is disruption of the vagus nerve branches. Second, anatomical gastrointestinal reconstruction [6, 7], gastrectomy, and lymphadenectomy, if needed for malignancy, could also have roles [1, 3, 8-12].

The major theoretical advantage of performing PC during gastric surgery is the avoidance of possible reintervention in the case of acute postoperative complications such as cholecystitis or common bile duct stones [13-16], especially for those patients who have to undergo 
multitherapeutic strategies (i.e., adjuvant chemotherapy) [17]. In these instances, both laparoscopic cholecystectomy and endoscopic retrograde cholangiography could be challenging, if not impossible, in the presence of previous reconstructions or adhesions [18, 19]. A recent article and retrospective review reported increased mortality rates after subsequent cholecystectomy [5, 20]. Conversely, PC achieved during open or minimally invasive surgery is not time-consuming and has minimal additional risks for patients [21-24]. However, there are many concerns regarding this strategy. First, most anecdotal reports of biliary complications after gastric surgery have been related to peptic diseases or, more recently, morbid obesity, and only a few surviving patients with cholelithiasis after gastric surgery for cancer are expected to develop symptoms or require additional treatments [2,3]. With improvements in surgical skills and technical equipment, the standard laparoscopic cholecystectomy is often feasible, even for those patients who have undergone the previous gastric surgery with minimal risks of conversion and bile duct injuries [18, 25, 26]. Finally, the increasing issues of litigation and malpractice claims have raised some concerns regarding the removal of a healthy organ.

Conversely, recent multimodal approaches have prolonged the survival of cancer patients, including those affected by gastric malignancy, thus increasing the possibility of gallstone development after surgery. Moreover, almost $90 \%$ of those who will develop calculi will do so within 2 years after surgery [3]. Therefore, with a 5-year survival rate of up to $50 \%$ at many centers $[27,28]$, a larger number of patients could be at risk for biliary complications during follow-up.

Unfortunately, no strong, statistically relevant, conclusive study has been reported in the literature, and most results are contrasting and of poor quality. Some authors $[3,8]$ have recommended performing $\mathrm{PC}$ at the time of gastric surgery to avoid complications and impairment of quality of life for surviving patients; however, others have not $[1,14]$. After discussing the opportunity for concomitant surgery with the patient, each surgeon is able to decide, according to preference, whether PC should be proposed on a routine basis if gallbladder calculi or biliary sludge is present.

The aim of the trial was to investigate if the PC performed during the standard surgery for gastric cancer had a clinical impact during the long-term follow-up.

\section{Methods}

\section{Trial design}

After formal approval by the ethics committee of each participating center, the Cholegas Study, a randomized, multicenter, controlled trial regarding PC during gastric surgery for cancer, began in Italy in November 2008 (Trial Registration: ClinicalTrials.gov ID. NCT00757640) [29]. The recruitment ended in March 2012 [30], and follow-up was concluded in March 2017. The aim was to investigate whether intervention was needed and advisable. The nine participating centers were distributed throughout Italy, and the surgeons (members of the GIRCG [Italian Research Group for Gastric Cancer]) had previously performed certified gastric surgery. Details of the study protocol have been published elsewhere [29]. In brief, the protocol required a complete preoperative data collection, including patient demographics and comorbidities, details of gastric surgery, perioperative treatment and complications, and follow-up. A routine ultrasonography of hepatobiliary region was scheduled after 4 months, thereafter every 6 months for the first 2 years and yearly for the rest of the follow-up (at least 5 years) to assess possible cholelithiasis development. Routine blood checks with specific hepatic tests were performed with the same schedule.

\section{Participants}

Inclusion criteria were age younger than 80 years and a histopathologically confirmed gastric adenocarcinoma. Those who underwent the previous cholecystectomy, those who had confirmed cholelithiasis or biliary sludge, and those who had metastases or some metabolic disease that may favor gallstone formation (i.e., hemolytic anemia or genetic hypercholesterolemia) were excluded. After each patient read a simplified abstract of the study, formal written consent to participate in the study was obtained.

Patients with confirmed cholelithiasis or with risk factors for biliary sludge underwent standard cholecystectomy on a routine basis, whereas other potentially eligible patients who refused to participate in the study underwent PC according to the surgeon's decision.

\section{Interventions}

According to a computer-generated list, eligible patients were randomized into two groups: PC during standard surgery (PC group) and standard gastric surgery only [standard surgery group (control group)]. The general oncologic principles of the standard gastric surgery (subtotal or total gastrectomy) were maintained, including vascular ligation and lymphadenectomy (D1, D2, and D3). No protocol restrictions regarding the use of minimally invasive technologies were required. Moreover, all types of reconstructions were allowed, including stapled or hand-sewn anastomosis.

Other technical details, such as the positioning of drainage and naso-gastric suction equipment, were used based on each surgeon's preference. If PC were required by 
randomization, then no particular technical recommendation was offered and any surgical devices, including clips and sutures, were allowed.

\section{Study endpoints}

The primary endpoint of the present study was cholelithiasis-free survival after gastrectomy for gastric adenocarcinoma, and the study was powered on this outcome. Cholelithiasis or biliary sludge was detected by ultrasound exam. Symptomatic cholelithiasis and cholecystectomy were also evaluated as a secondary endpoint of efficacy. In addition, a short-term endpoint of safety (complication rate, operative time, and postoperative stay) was addressed in a previous article [30].

An interim analysis to assess the safety of PC during gastric surgery was required by the ethics committee of the leading center and published in abstract form after half of the planned enrollment had concluded (sixty patients).

\section{Statistical analyses}

Sample size calculations and relevant statistical assumptions were reported along with the trial protocol [29]. We assumed that patients undergoing gastrectomy plus PC would not have experienced biliary disorders 5 years later and that $20 \%$ of those undergoing standard surgery would have experienced it. With a planned accrual period of 2 years, a minimum follow-up period of 3 years, and $50 \%$ of patients lost to follow-up (deceased or untraced) after 3 years, at least 122 patients (61 in each group) were considered necessary to ensure $80 \%$ power with a two-sided alpha of $5 \%$.

Significant differences between the PC and standard surgery groups were evaluated by the non-parametric Wilcoxon-Mann-Whitney rank-sum test for quantitative variables and by Fisher's exact test for categorical variables.

As planned in the study protocol [29], the incidence of cholelithiasis was compared between the control and treated groups by survival curves, estimated using the Kaplan-Meier method. Ultrasound detection of gallbladder calculi or other biliary abnormalities was considered as terminal event and death from any cause as censoring event. Significance of differences was evaluated by the logrank test when the hazard functions were proportional over time, and by the Wilcoxon-Breslow-Gehan test otherwise. A subgroup analysis, not initially planned in the study protocol, was added before seeing long-term results, and involved patients with pathological reports of the early stages [American Joint Committee on Cancer (AJCC) stage I or II].

Demographic characteristics, perioperative records, and data collected after the conclusion of the follow-up were electronically sent from each participating center to the leading center using a standard, simple, presettled database
(Microsoft Access; Microsoft Corporation Italia, Segrate, Milan, Italy). Analyses were performed using Stata statistical software (release 14; StataCorp, College Station, TX, USA), and statistical significance was set at $<0.05$.

\section{Results}

\section{Patients}

The Cholegas Study recruitment ended in 2012. One hundred and sixty-two patients from nine participating centers were randomized. The final analysis included $130(80.2 \%)$ patients. Details regarding randomization, inclusion and exclusion criteria, and reasons why some eligible and randomized patients dropped out have been published elsewhere [30] and are summarized in Fig. 1. Despite no technical restriction was included in planned protocol, no laparoscopic gastrectomy was collected.

The final analysis included 65 patients who underwent PC and 65 who did not [standard surgery group (control group)]. The two groups were comparable regarding demographic characteristics, body mass index, and definitive pathological staging (Table 1).

Variables related to the surgical technique were superimposable in the two groups. Median duration of surgery was 210 min in both controls (120-345) and patients undergoing PC (140-350); likewise, median blood loss was $200 \mathrm{ml}$ in both groups (100-900 and 50-1000 ml, respectively). Only one postoperative death occurred in the intervention group (1.5\%). The overall complication rates were $25 \%$ for the intervention group and $17 \%$ for the control treatment. None of these differences were statistically significant.

Only one (1.5\%) biliary complication was recorded for the PC group during the postoperative period. The patient experienced biliary leakage during drainage; however, it spontaneously resolved within a few days (Clavien-Dindo 1). No definitive conclusion was achieved regarding the origin of the leak; however, the most likely candidates were the gallbladder site and the bile ducts or a small duodenal dehiscence. Although a direct analysis of the incidence of biliary injuries was not a target of the protocol, the safety of concomitant cholecystectomy during gastric surgery for cancer was assessed and presented elsewhere [30].

\section{Overall survival}

The planned study protocol [29] required a minimum follow-up of 3 years for the last patient included. The median follow-up for surviving patients was 61 months (interquartile range, 47-69 months).

Overall survival did not differ between the control and treated groups: 5-year overall survival was $60 \%$ for the 


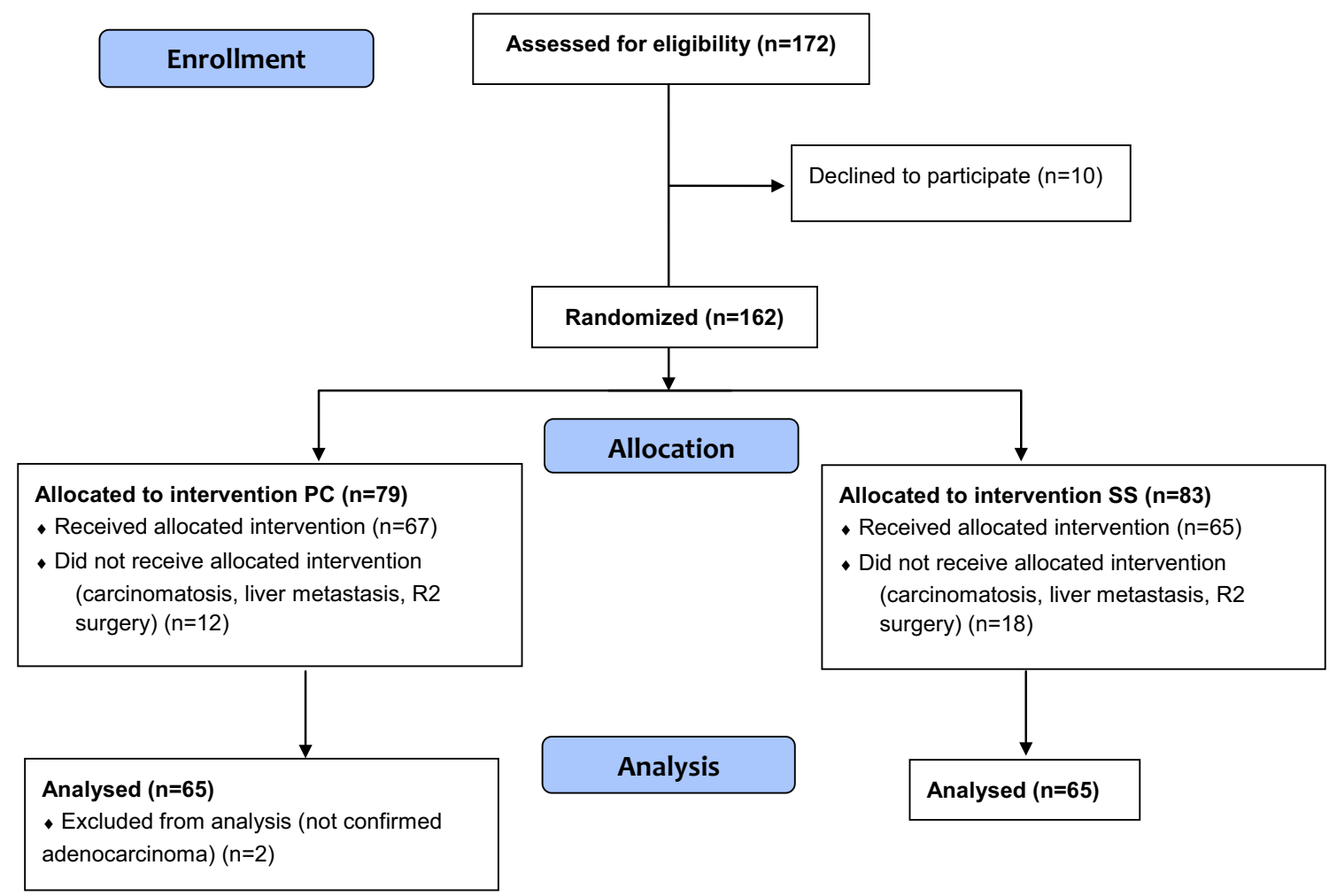

Fig. 1 CONSORT flowchart showing number of included patients during the study and the reasons for loss to final follow-up. $P C$ prophylactic cholecystectomy, $S S$ standard surgery

control group (95\% CI $47-71 \%)$ and $59 \%$ for the group undergoing PC (95\% CI 44-71\%) (log-rank test: $p=0.697$ ). Likewise, no survival difference between the control and treated groups was observed when considering only the early stages (AJCC stages I or II). The 5-year overall survival was 77\% (59-88\%) for the control group and 76\% (95\% CI $57-87 \%$ ) for the treated group (log-rank test: $p=0.864$ ).

\section{Biliary abnormalities}

During follow-up, eight patients (12.3\%)—four with gallbladder calculi and four with biliary sludge-in the standard surgery group and one (1.5\%)—abnormal main duct dilatation-in the PC group, developed some kind of biliary abnormality ( $8 / 65$ vs. $1 / 65, p=0.033)$. Among these patients, only three $(4.6 \%$, reflecting $33 \%$ of the "biliary abnormalities") in the standard surgery group and none in the intervention group were symptomatic ( $3 / 65$ vs. $0 / 65$, $p=0.244)$. One of these three patients underwent cholecystectomy during follow-up; another was scheduled for the same surgery but had not undergone the procedure at the time of data collection; the third patient developed acute pancreatitis of biliary origin, which resolved with medical therapy. Therefore, the number needed to treat with PC to avoid reoperation for cholelithiasis was 1:32.5. The group treated with PC had none of these disturbances.

When considering patients who were still alive after 60 months of follow-up, the cumulative incidence of cholelithiasis or biliary sludge increased to $18.5 \%$ (5/27) for the control group, while no further case was recorded in the PC group.

The difference in the crude number of ultrasound-detected abnormalities, highlighted by the Fisher's exact test, was not confirmed when time to abnormality onset was taken into account. Cholelithiasis-free survival curves, computed considering ultrasound detection of biliary abnormalities as the terminal event, did not statistically differ between the two groups (Wilcoxon-Breslow-Gehan test: $p=0.267$ ) (Fig. 2). Moreover, the two groups did not significantly differ even when the survival analysis focused on the late part of follow-up, i.e., after 48 months (Wilcoxon test; $p=0.143$ ). The same pattern was recorded during the subgroup analysis of patients with a pathological report of the early stages (AJCC stage I or II) (Fig. 3).

Finally, an attempt to identify any risk factors for biliary abnormalities was performed for the standard surgery group. Those who had positive sonography results were compared with those who did not according to age, sex, body mass index, type of surgery (total gastrectomy or 
Table 1 Comparison of patients' characteristics, details of interventions, and tumor stage between the groups undergoing PC or standard gastric surgery only

\begin{tabular}{|c|c|c|c|}
\hline & $\begin{array}{l}\text { Prophylactic } \\
\text { cholecystectomy } \\
(n=65)\end{array}$ & $\begin{array}{l}\text { Standard } \\
\text { surgery } \\
(n=65)\end{array}$ & $p$ value $*$ \\
\hline Age (years) & $67(24-80)$ & $69(43-80)$ & 0.89 \\
\hline $\operatorname{Sex}(\mathrm{F} / \mathrm{M})$ & $27 / 38$ & $28 / 37$ & 1.00 \\
\hline BMI $\left(\mathrm{kg} / \mathrm{m}^{2}\right)$ & $24(19-37)$ & $24(18-37)$ & 0.28 \\
\hline Type of gastrectomy & & & 0.46 \\
\hline Subtotal & $46(71)$ & $41(63)$ & \\
\hline Total & $19(29)$ & $24(37)$ & \\
\hline Lymphadenectomy & & & 0.86 \\
\hline D1 & $5(8)$ & $7(11)$ & \\
\hline D2 & $52(80)$ & $50(77)$ & \\
\hline D3 & $8(12)$ & $8(12)$ & \\
\hline Reconstruction & & & 0.43 \\
\hline Roux-en-Y & $45(69)$ & $50(77)$ & \\
\hline Billroth II & $20(31)$ & $15(23)$ & \\
\hline Tumor stage (pTNM) & & & 0.75 \\
\hline Stage I & $25(38)$ & $25(38)$ & \\
\hline Stage II & $6(10)$ & $7(11)$ & \\
\hline Stage III & $32(49)$ & $33(51)$ & \\
\hline Missing data & $2(3)$ & $0(0)$ & \\
\hline
\end{tabular}

Continuous variables are expressed as median and range; categorical data as number and percentage

BMI Body Mass Index, $p T N M$ pathological tumor node metastasis (7th ed)

* $p$ values were computed by Wilcoxon-Mann-Whitney rank-sum test for quantitative variables and by Fisher's exact test for categorical variables

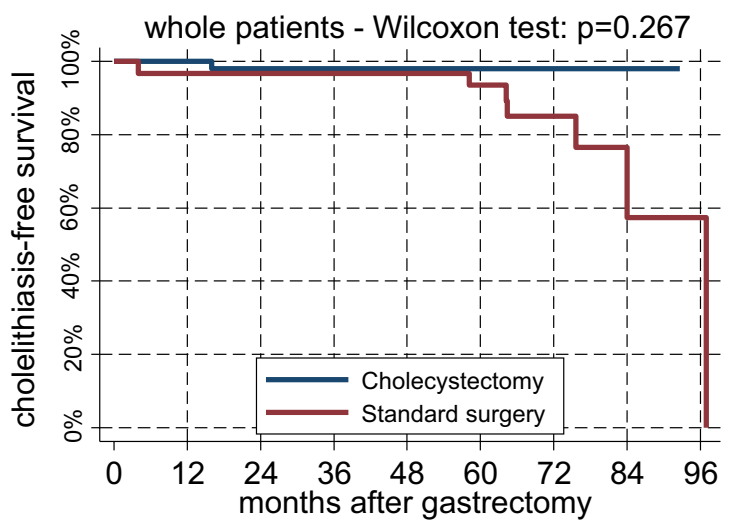

Number at risk

$\begin{array}{lllllllcll}\text { Cholecystectomy } & 65 & 50 & 44 & 37 & 28 & 18 & 8 & 3 & 0 \\ \text { Standard surgery } & 65 & 52 & 43 & 38 & 32 & 27 & 14 & 4 & 1\end{array}$

Fig. 2 Cholelithiasis-free survival curves in all patients, estimated by Kaplan-Meier method. "Ultrasound appearance" of biliary disorder was considered as terminal event and death from any cause as censoring event

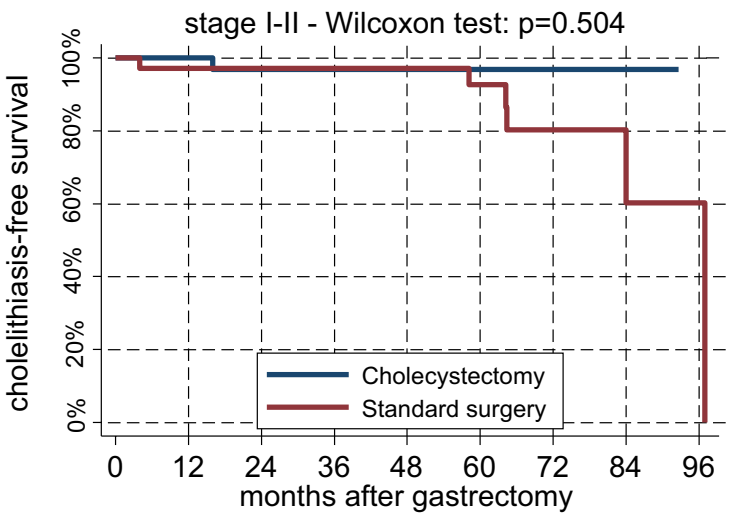

Number at risk

$\begin{array}{llllllllll}\text { Number at risk } & & & & & & \\ \text { Cholecystectomy } & 37 & 31 & 30 & 27 & 21 & 15 & 6 & 3 & 0 \\ \text { Standard surgery } & 36 & 33 & 31 & 28 & 23 & 19 & 9 & 4 & 1\end{array}$

Fig. 3 Cholelithiasis-free survival curves in the less advanced tumor stages, estimated by Kaplan-Meier method. "Ultrasound appearance" of biliary disorder was considered as terminal event and death from any cause as censoring event

Table 2 Comparison between patients who developed "biliary abnormalities" at sonography in the Standard Surgery group (without prophylactic cholecystectomy) (ECHO 1) and those who did not (ECHO $0)$

\begin{tabular}{llll}
\hline & ECHO 1 $(n=8)$ & ECHO 0 $(n=57)$ & $p$ value* \\
\hline Age (years) & $71(55-75)$ & $70(60-76)$ & 0.982 \\
BMI $\left(\mathrm{kg} / \mathrm{m}^{2}\right)$ & $24(21-25)$ & $24(22-26)$ & 0.541 \\
Sex & & & 0.717 \\
Women & $4(14)$ & $24(86)$ & \\
Men & $4(11)$ & $33(89)$ & \\
Type of surgery & & & 0.471 \\
Total gastrectomy & $2(8)$ & $23(92)$ & \\
Subtotal gastrectomy & $6(15)$ & $34(85)$ & \\
Type of reconstruction & & & \\
Roux-en-Y & $6(12)$ & $43(88)$ & \\
Billroth II & $2(12.5)$ & $14(87.5)$ & \\
Lymphadenectomy & & & \\
D1 & $1(17)$ & $5(83)$ & \\
D2 & $6(12)$ & $46(88)$ & \\
D3 & $1(14)$ & $6(86)$ & \\
\hline
\end{tabular}

Continuous variables are expressed as median and interquartile range; categorical variables are expressed as number and percentage

$B M I$ Body Mass Index

* $p$ values were computed by Wilcoxon-Mann-Whitney rank-sum test for quantitative variables and by Fisher's exact test for categorical variables

subtotal gastrectomy), surgical reconstruction (Roux-en-Y and Billroth II), and extension of lymphadenectomy (D1, D2, or D3). None was significantly associated with biliary abnormalities (Table 2). 


\section{Discussion}

The present study was the first randomized, controlled trial comparing PC during gastric surgery for cancer to the standard treatment (no concomitant cholecystectomy). The trial showed that the primary endpoint, i.e., cholelithiasis-free survival, was not significantly modified by the experimental intervention.

The trial design [29] investigated the differences between the two groups but was not intended to measure the risk of biliary injuries during concomitant cholecystectomy, because that would have required many more patients. However, the local ethics committee of the leading center required an interim analysis of the safety of PC during gastric surgery, which was very unusual because of its widespread use in the global surgical community.

Demographic perioperative outcomes and pathological findings were comparable between the two groups according to the blind randomization protocol [30] and were consistent with Italian and international gastric cancer surgery outcomes [28, 31-35]. One limitation to extending our results to an eastern series was the routine use of reconstructions using only Roux-en-Y and Billroth II; Billroth I is rarely used in western countries [36].

Interestingly, performing $\mathrm{PC}$ during gastric cancer surgery required no significant extra time. No other surgical or non-surgical complications were directly related to cholecystectomy itself; therefore, morbidity was equally distributed between the two study groups [30].

Although the mortality risk was the highest during the first 3 years after gastrectomy [37], nearly all biliary abnormalities found in the control group were sonographically detected after 4.5 years. Moreover, the cumulative incidence of cholelithiasis or biliary sludge increased to $18.5 \%(5 / 27)$ when considering only patients who were still alive 60 months after surgery. On the contrary, cholelithiasis was not observed after PC. During ultrasound, only one patient presented abnormal biliary duct dilatation without abnormal blood test results, symptoms of biliary disease, or the need for further treatments.

However, symptomatic cholelithiasis was rather rare, affecting only three patients (4.6\%) in the control group (33\% of the diagnosticated "biliary abnormalities"), this reflecting similar findings in the general population. Nevertheless, most of the epidemiologic studies regarding symptomatic patients included "gallstones disease" rather than the wider spectrum of "biliary abnormalities" or "biliary sludge" [38]. In addition, a symptomatic gallbladder is difficult to define, because its clinical presentation overlaps with that of other postgastrectomy syndromes [39].

Although, in the present clinical trial, PC significantly reduced the crude number of biliary abnormalities after gastric surgery, the difference between the treated and control groups was not significant in the preplanned survival analysis. This apparent discrepancy was mainly due to delayed onset of biliary abnormalities: in the control group, 6 abnormalities out of 8 were sonographically detected after 4.5 years from gastrectomy, so that survival curves started to diverge thereafter. Moreover, although the experimental procedure prevented the onset of biliary symptoms in the treated group, these outcomes were rare even in the control group, so that the difference was not statistically significant. Hence, PC lacked a clinical advantage.

These findings are consistent with an impressive review of concomitant cholecystectomy during gastro-esophageal surgery. The authors concluded that less than $5 \%$ of surgical patients will require subsequent cholecystectomy, and most of these procedures will be performed laparoscopically and with few additional morbidity and mortality risks [14]. Nevertheless, another study including more than 17,000 gastric cancer patients reported an incidence $7.4 \%$ of cholelithiasis during follow-up; half of them required further surgery, and the mortality rate related to the second procedure was $1.8 \%$ [5]. However, according to the present study, prophylactic surgery was not justified if the development of symptoms or late cholecystectomy was the only negative clinical endpoints.

Some limitations should be acknowledged in the present study. Although the follow-up was adequate (median 62 months), our sample size was smaller than those reported by others [5], thus limiting the power to detect some specific outcomes. Sample size had been planned to investigate ultrasound-detected biliary abnormalities. Hence, the secondary outcome, "symptomatic cholelithiasis" $(n=3)$ or reintervention $(n=2)$, presented such low incidence that it could not be studied in depth. In addition, our study had not enough power to detect possible differences in risk factors when comparing patients who developed ultrasound biliary abnormalities to those who did not. A recent study published by Liang et al. [5] that involved more than 17,000 gastric cancer patients identified important risk factors for the development of biliary complications. In multivariable analysis, older age, total gastrectomy, duodenal exclusion, diabetes, cirrhosis, and other comorbidities contributed to biliary disease. In addition, younger patients affected by symptomatic cholelithiasis or those who required readmission were at risk for the subsequent surgery. However, the results of that study did not conflict with our results for two main reasons. First, the study designs were completely different regarding the populations, aims, and statistical approach. Second, the results of the study by Liang [5] could be integrated with and completed by ours, thereby permitting some definite conclusions regarding the subgroup of patients who would benefit from PC. 
In conclusion, concomitant PC during gastric surgery for malignancies is safe, although not effective for improving the natural course of patients. Indeed, although the additional intervention prevented the development of symptomatic biliary calculi or sludge in the long term, these biliary abnormalities were mostly asymptomatic and delayed. Moreover, according to the present trial, the number needed to treat to avoid reoperation for cholelithiasis was 1:32.5, and such a ratio does not allow for PC as routine practice. A more rational approach could be to consider the procedure for patients at higher risk for cholelithiasis, such as younger patients with the early gastric cancer whose life expectancy is high.

Acknowledgements Italian Research Group for Gastric Cancer (GIRCG): Other surgeons who participated in the study were Marco Bernini, MD, PhD (Surgical Oncology, Careggi University Hospital, Florence, Italy), Leonardo Gerard, MD (General Surgery, Ospedale Carlo Poma, Mantova, Italy), Renato Moretti (Surgical Oncology, Careggi University Hospital, Florence, Italy), Luca Cozzaglio, MD (Division of Surgical Oncology, Humanitas Hospital, Milan, Italy), Stefano Berardi, MD (Surgical Oncology Catholic University, Campobasso, Italy), and Paolo Bechi (General Surgery, Careggi University Hospital, Florence, Italy).

Funding The native English-speaking translation was supported by the funds of the University of Florence, Italy.

\section{Compliance with ethical standards}

Conflict of interest The authors have no conflicts of interest to declare.

Ethical approval A formal approval by the ethics committee of each participating center was obtained before the trial started recruitment.

\section{References}

1. Kobayashi T, Hisanaga M, Kanehiro H, et al. Analysis of risks factors for the development of gallstones after gastrectomy. Br J Surg. 2005;92:1399-403.

2. Sakorafas GH, Milingos D, Peros G. Asymptomatic cholelithiasis: is cholecystectomy really needed? Dig Dis Sci. 2007;52:1313-25.

3. Fukagawa T, Katai H, Saka M, et al. Gallstone formation after gastric cancer surgery. J Gastrointest Surg. 2009;13:886-9.

4. Li VKM, Pulido N, Martinez-Suartez P, et al. Symptomatic gallstones after sleeve gastrectomy. Surg Endosc. 2009;23:2488-92.

5. Liang TJ, Liu SI, Chen YC, et al. Analysis of gallstone disease after gastric cancer surgery. Gastric Cancer. 2017;20:895-903.

6. Inoue K, Fuchigami A, Higashide S, et al. Gallbladder sludge and stone formation in relation to contractile function after gastrectomy. Ann Surg. 1992;215:19-26.

7. Qvist N. Review article: gall-bladder motility after intestinal surgery. Aliment Pharmacol Ther. 2000;14(s2):35-8.

8. Wu CC, Chen CY, Wu TC, et al. Cholelithiasis and cholecystitis after gastrectomy for gastric carcinoma: a comparison of lymphadenectomy of varying extent. Hepatogastroenterology. $1995 ; 42: 867-72$

9. Tomita R, Tanjoh K, Fujisaki S. Total gastrectomy reconstructed by interposition of a jejunal $\mathrm{J}$ pouch with preservation of hepatic vagus branch and lower esophageal sphincter for T2 gastric cancer without lymph node metastasis. Hepatogastroenterology. 2004;51:1233-40.

10. Akatsu T, Yoshida M, Kubota T, et al. Gallstone disease after extended (D2) lymph node dissection for gastric cancer. World J Surg. 2005;29:182-6.

11. Paik KH, Lee JC, Kim HW, et al. Risk factors for gallstone formation in resected gastric cancer patients. Medicine (Baltimore). 2016;95:e3157.

12. Tyrväinen T, Nordback I, Toikka J, et al. Impaired gallbladder function in patients after total gastrectomy. Scand J Gastroenterol. 2017;52:334-7.

13. Oh SJ, Choi WB, Song J, et al. Complications requiring reoperation after gastrectomy for gastric cancer: 17 years experience in a single institute. J Gastrointest Surg. 2009;13:239-45.

14. Gillen S, Michalski CW, Schuster T, et al. Simultaneous/Incidental cholecystectomy during gastric/esophageal resection: systematic analysis of risks and benefits. World J Surg. 2010;34:1008-14.

15. Liu XS, Zhang Q, Zhong J, et al. Acute cholecystitis immediately after radical gastrectomy: a report of three cases. World J Gastroenterol. 2010;16:2702-2074.

16. Oida T, Kano H, Mimatsu K, et al. Cholecystitis or cholestasis after total gastrectomy and esophagectomy. Hepatogastroenterology. 2012;59:1455-7.

17. Jayakrishnan TT, Groeschl RT, George B, et al. Review of the impact of antineoplastic therapies on the risk for cholelithiasis and acute cholecystitis. Ann Surg Oncol. 2014;21:240-7.

18. Sasaki A, Nakajima J, Nitta H, et al. Laparoscopic cholecystectomy in patients with a history of gastrectomy. Surg Today. 2008;38:790-4.

19. Fraser SA, Sigman H. Conversion in laparoscopic cholecystectomy after gastric resection: a 15-year review. Can J Surg. 2009;52:463-6.

20. Miftode SV, Troja A, El-Sourani N, et al. Simultaneous cholecystectomy during gastric and oesophageal resection: a retrospective analysis and critical review of literature. Int J Surg. 2014;12:1357-9.

21. Juhasz EZ, Wolff BG, Meagher AP, et al. Incidental cholecystectomy during colorectal surgery. Ann Surg. 1994;219:467-74.

22. Wolff BG. Current status of incidental surgery. Dis Colon Rect. 1995;38:435-41.

23. Watemberg S, Landau O, Avrahami R, et al. Incidental cholecystectomy in the over-70 age group. A 19-year retrospective, comparative study. Int Surg. 1997;82:102-4.

24. Murata A, Okamoto K, Muramatsu K, et al. Effects of additional laparoscopic cholecystectomy on outcomes of laparoscopic gastrectomy in patients with gastric cancer based on a national administrative database. J Surg Res. 2014;186:157-63.

25. Kwon AH, Inui H, Imamura A, et al. Laparoscopic cholecystectomy and choledocholithotomy in patients with a previous gastrectomy. J Am Coll Surg. 2001;193:614-9.

26. Joohyun K, Jeong NC, Sun HJ, et al. Multivariable analysis of cholecystectomy after gastrectomy: laparoscopy is a feasible initial approach even in the presence of common bile duct stones or acute cholecystitis. World J Surg. 2012;36:638-44.

27. Lepage C, Sant M, Verdecchia A, et al. EUROCARE working group. Operative mortality after gastric cancer resection and long-term survival differences across Europe. Br J Surg. 2010;97:235-9.

28. Marrelli D, Pedrazzani C, Morgagni P, et al. on behalf of the Italian Research Group for Gastric Cancer (GIRCG). Changing clinical and pathological features of gastric cancer over time. $\mathrm{Br}$ J Surg. 2011;98:1273-83.

29. Farsi M, Bernini M, Bencini L, et al. GIRCG (Gruppo Italiano di Ricerca sul Cancro Gastrico). The CHOLEGAS study: multicentric randomized, blinded, controlled trial of gastrectomy plus 
prophylactic cholecystectomy versus gastrectomy only, in adults submitted to gastric cancer surgery with curative intent. Trials. 2009;15:10-32.

30. Bernini M, Bencini L, Sacchetti R, et al. Italian Research Group for Gastric Cancer (IRGGC). The Cholegas Study: safety of prophylactic cholecystectomy during gastrectomy for cancer: preliminary results of a multicentric randomized clinical trial. Gastric Cancer. 2013;16:370-6.

31. Enzinger PC, Benedetti JK, Meyerhardt JA, et al. Impact of hospital volume on recurrence and survival after surgery for gastric cancer. Ann Surg. 2007;245:426-34.

32. Hartgrink HH, Jansen EP, van Grieken NC, et al. Gastric cancer. Lancet. 2009;374:477-90.

33. Kodera E, Fujiwara M, Ito Y, et al. Radical surgery for gastric carcinoma: it is not an issue of whether to perform D1 or D2. Dissect as many lymph nodes as possible and you will be rewarded. Acta Chir Belg. 2009;109:27-35.
34. Shi Y, Zhou Y. The role of surgery in the treatment of gastric cancer. J Surg Oncol. 2010;101:687-92.

35. Saka M, Morita S, Fukagawa T, et al. Present and future status of gastric cancer surgery. Jpn J Clin Oncol. 2011;41:307-13.

36. Kimura J, Kunisaki C, Takagawa R, et al. Is routine prophylactic cholecystectomy necessary during gastrectomy for gastric cancer? World J Surg. 2017;41:1047-53.

37. Verlato G, Marrelli D, Accordini S, et al. Short-term and longterm risk factors in gastric cancer. World J Gastroenterol. 2015;21:6434-43.

38. Abraham S, Rivero HG, Erlikh IV, et al. Surgical and nonsurgical management of gallstones. Am Fam Physician. 2014;89:795-802.

39. Karanicolas PJ, Graham D, Gönen M, et al. Quality of life after gastrectomy for adenocarcinoma: a prospective cohort study. Ann Surg. 2013;257:1039-46. 